\title{
RECOMMENDER SYSTEM USING UNSUPERVISED MACHINE LEARNING FOR SATISFACTION SURVEYS
}

\author{
Baba Mbaye \\ EFFET B company, France \\ Laboratory of ELLIADD, University of Franche-comtè, France
}

\begin{abstract}
Satisfaction surveys are being used more and more by companies to improve their sales force. With the development of new technologies the piloting of these satisfaction surveys is digitized in a partial way.

Piloting these surveys often involves the expertise of a human agent in order to make a judgment on the results obtained from satisfaction surveys. This is a tedious task for the decision-maker, as it faces a huge and heterogeneous amount of data.

This problem may be mitigated by using a recommendation engine based on the unsupervised machine learning algorithm. This recommendation system (RS) will be oriented towards two axes: decision-making (DM) and machine learning (ML).

In our approach, we use RS for consistency between the user and the recommended items. ML will allow us to include in our list of recommendations, unexpected items, items that are not derived from the algorithmic logic of the recommendation system and to make the system partially autonomous on decision-making (to less involving the recommendation engine). Our approach is divided into a) the recommendation process for decision-making, b) unsupervised ML and c) partial "empowerment" for decision-making.
\end{abstract}

\section{KEYWORDS}

Recommender System, Unsupervised Machine Learning, Collaborative Filtering, Satisfaction Surveys

\section{INTRODUCTION}

Faced with the exponential growth of the amount of data from satisfaction surveys, experts are drowned in a mass of information that makes decision-making difficult in the face of a given problem. Various RS are often offered through research in various fields. The purpose of these RS is to help users find new items or services such as music, books or even people, based on user or item information (Abdi, H. 20007 ). These systems play a very important role in decision-making. Indeed, these allow us to maximize user profits (Chen, L. Et al, 2008) or to minimize risks (Bouneffouf et al, 2013).

These recommendation engines are built around a set of concepts and rules with results deductible most often by the human. By integrating ML algorithms, the system, over time, will allow us to obtain recommendations that we never thought (not deduction from the rules and concept from our recommendation engine).

In this paper we present a modeling of an RS using unsupervised ML. This recommendation system is of the collaborative filtering $(\mathrm{CF})$ type.

In our approach the ML is used for the purpose of obtaining results that are not derived from the concepts and rules of SR. Indeed, the ML adapts to the environment of execution of the algorithms and generates the appropriate models.

Thus, in this document we will first explain our contribution, then review the literature and present our approach to modeling the system. 


\section{CONTRIBUTION}

This work makes a significant contribution to the following fields of research: satisfaction surveys and SRs. First, we will review the literature on RSs, in their generalities and for satisfaction surveys. Then secondly, we will show the use of an unsupervised ML module combined with the RS module can generate more relevant, personalized and unintended recommendations. Thus our architecture will be composed of two parts : a motor part of recommendation and a second part which represents the module of ML a result, we will see how ML can improve cold start for a new user in a collaboratif filtering algorithm. We will also see how an ML system can be efficient and optimal by combining it with a recommendation engine. Finally, we show how unsupervised ML agorithms can lead us to obtain unexpected predictions. This study is applied with data from satisfaction surveys. A satisfaction survey is a process used by a company to evaluate the quality of its services.

\section{RELATED WORK}

\subsection{RS for Satisfaction Surveys}

The aim of an RS is to provide users with the resources required based on their credentials. The latter thus see their research time reduced, but also receive proposals from the system that they would not spontaneously pay attention to. The development of the web has greatly contributed to the establishment of such systems as in the field of e-commerce. RS can be viewed initially as a response to users who have difficulty making decisions in the context of using a "conventional" information retrieval system.

The research in information is based on a principle of indexing data in order to respond to requests from users. More specifically, documentary research, under the discipline of information retrieval, consists of querying a knowledge base through written queries in natural languages or in the form of key words (Joachim Schöpfel et al, 2017). These key words are then compared to all the indexes of the documents present in the database of the search engine when users redirect to a set of relevant results with their initial query (Hulusi Karagel et al 2014).

A satisfaction survey (mystery survey) is a point-of-sale visit by an investigator. The purpose of a visit to a point of sale is to measure the quality of the welcome, the quality of the advice and the respect of the sales instructions.(Yong Li et al, 2017).

These surveys are conducted by "fake" clients recruited and commissioned by companies to evaluate their sales network and are used in many economic sectors (e.g. Automotive, Banks, public energy regulators). They allow companies to acquire a concrete measure of the quality of a service offered in order to improve it, by modifying their offer or by offering training to their employees. Mystery surveys are complementary to satisfaction surveys that provide a measure of opinion as well as complaints denouncing possible malfunctions (AMF, 2011).

The purpose of these mystery visits is to reinforce preventive action in the area of savings protection by monitoring, in the field, the concrete conditions for marketing financial products accessible to the general public.

These mystery visits are a new tool enabling companies to identify as early as possible possible malfunctions in the marketing chain for financial products. The latter make it possible to highlight good and bad practices in the marketing of financial products and to detect the risks of abusive or inappropriate sales of financial products.

The recommendations made from satisfaction survey results are of the action plan type or of a personalized training list for the employees of the company that requested this survey.

\subsection{RS for Decision-Making (DM)}

According to (Woodman et al, 1976) "The decision is the best choice for a multitude of opinions in order to arrive at an optimal solution and a given problem". This definition reveals that decision-making is done in a collective way, from where it is necessary a good interaction between the different agents of the process.

Thus, the use of a CF type RS responds to this task. The CF is an approach based on the sharing of 
opinions between users. It reiterates the principle of "word of mouth" practiced by humans since always to build an opinion on a product or service they do not know (schafer et al., 2007). The principle of this method is that the opinions of other users are often used to provide a reasonable prediction of the active user's preference on an item he has not yet noted. These methods assume that if users have the same preferences on one set of items, then they will probably have the same preferences on another set of items they have not yet rated. Suppose for example that Mary's neighbors find that the new restaurant that is open in her neighborhood is a success, she may find it interesting to go try it. If, on the contrary, the majority of her neighbors feel that it is a failure, then she may decide to abstain from going. The techniques of CF therefore recommend to the current user, the items appreciated by the users with whom he shares the same tastes, we speak of similar users.

In our study, all items represent the list of action plans and training proposed by the system on confirmation of the expert. Indeed, the satisfaction survey allows the company that asked for it, first to obtain notes on the various vectors of satisfaction that it has set. These satisfaction vectors are modeled as questions that constitute the questionnaire of the satisfaction survey.

\section{$3.3 \mathrm{ML}$}

Machine learning is increasingly used in intelligent software (Simon et al, 2018). It is a subfield of artificial intelligence that focuses on the development of models able to represent certain characteristics of the world around us, to learn some statistical properties of the distributions of the data they process, with the aim of accomplish a multitude of tasks (rem N. Ramkumar et al, 2018). Its link with intelligence is derived from the ability of these models to generalize, extract relevant information from data processed during an update process called "training" and to reuse them effectively on new data never previously encountered. A model is a decision function that in the case of supervised learning takes as input a value $x \in \mathbb{R}$ et $\left\{\left(\mathrm{x}_{\mathrm{i}}, \mathrm{y}_{\mathrm{i}}\right)\right\}_{\mathrm{i}=1}^{\mathrm{n}}$ where $y_{i}$ is the target value associated with $x_{i}$ and qui returns a prediction $f\left(x_{i}\right)$ mean of $y_{i}$. When the target is discrete, we are talking about a classification task (Yanxin Zhang et al 2019).

In unsupervised learning, there are no explicit targets. We can take an example of an estimate on the reading time of a document, for example, or the probability of rainfall.

In the case of recommendation systems, ML is a solution for predicting recommendations that the expert does not even think about. The data provided to the artificial intelligence system is not tagged or categorized, and the system's algorithms process the data without any prior training, the results obtained at the output depend on the algorithms implemented (Ezequiel López-Rubio et al, 2018). Unsupervised learning algorithms can perform more complex processing tasks than supervised learning systems, but they can also be unpredictable (Gangadhar Shobha et al , 2018).

Even if an unsupervised ML manages to sort out shirts and Tshirts for example, it can also add unexpected and unwanted categories to classify for example brands of unusual shirts and t-shirts creating confusion instead of putting on order.

Unsupervised machine learning algorithms organize the data into a group of clusters. It describes its structure and gives complex data a simple, organized look for analysis (Ezequiel López-Rubio et al, 2018).

Unsupervised learning occurs when you do not have labeled data for training. This is the basic type algorithm where you only have input data and no output variables that coincide. These are called this because there is no corresponding output for a particular input. Their problems can be grouped together in a cluster to discover the inherent clustering and association issues. Some popular examples of unsupervised learning algorithms are k-ways for grouping problems used in referral systems, customer segmentation, targeting marketing, and the dimensionality reduction problem for data visualization. bulky, feature development, structure discovery, etc (Ezequiel López-Rubio et al, 2018).

ML is a category of algorithms that allows applications to make accurate predictions without explicit programming.

\subsection{RS using ML}

Nowadays, RSs are used in many information-based companies, such as facebook (Tadlaoui, 2015), Google (Liu et al 2010), or Netflix (Steck at al, 2013). The field of SR came into being in the mid-1990s with the introduction of Tapestry (Goldberg et al, 1992), the first RS. As the RS field evolved, researchers studied the use of algorithms derived from machine learning (ML), an area of artificial intelligence (AI). Machine Learning has been studied since the late 1950s (Martens et al, 1959), with the emergence of the AI field. There is now a plethora of ML algorithms (k-nearest neighbor (Walid, 2018), clustering (Jinhong Huang et al, 
2018), Bayes network (Friedman et al, 1997), to name just a few. some types, used in applications ranging from pattern recognition in images (Emiliano et al, 2019) to autonomous vehicles (Nynke et al, 2017). The potential application of ML algorithms is vast and the field looks very promising.

This ML can be of three types: supervised, unsupervised or reinforcement learning.

In the case of recommendation systems built on unsupervised learning personalize the delivery of online advertisements in real time. It's no coincidence that a search on amazon brings up shoe ads on other websites.

\section{OUR APPROACH AND EMPOWERMENT OF DM}

As a computer domain, machine learning is closely related to mathematical statistics; Having a through knowledge of statistics is useful for understanding and exploiting ML algorithms (Samuel Eisenberg et al 2018).

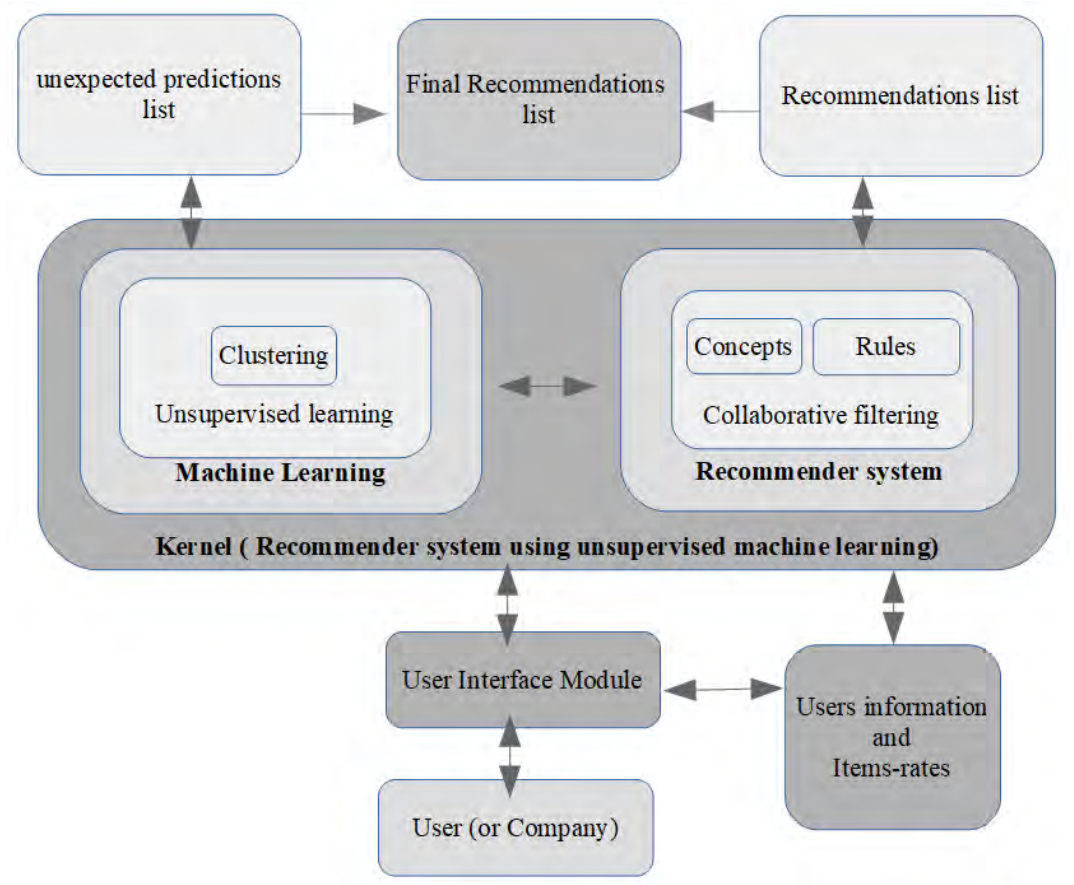

Figure 1. Kernel of recommender system using unsupervised machine learning

So in our recommendation system we use unsupervised learning algotrihms. These unsupervised ML algorithms will allow us to obtain unexpected predictions, ie recommendations that differ from those from a set of predefined rules and concepts in the recommendation engine.

Our architecture will consist of a core containing two modules. A first module representing our RS by collaborative filtering and a second module where ML algorithms will be modeled.

The idea here is to do at the same time, two preliminary recommendations process treatments, one by collaborative filtering based on a set of rules and concepts and the other by a machine learning engine. This learning engine will take into account the conditions that are not applicable to the predefined rules in the collaborative filtering algorithm. Indeed the unsupervised ML algorithms will take into account other additional factors for the construction of these models of recommendations. The factors are : the recommendation context, scalability, overall modeling, automation and iterative processes. The items from these two recommendations will be ranked in order of relevance according to user profile. To do this we apply these items in a Bayesian classification algorithm on a predefined model model.

For a new user (a new company in our case study), we assume that the ML can mitigate cold start. Indeed, by using a system with unsupervised learning, the system can build a model from the cold data of the user and the distance of similarity with the users who have already received recommendations in the past. As a result, from this model the new user will be recommended items of acceptable relevance.

This below, we present you, our system architecture of RS netwire by filtering collabotafi using the ML. 


\section{CONCLUSION}

In this article we presented a modeling of a recommendation system using unsupervised machine learning for satisfaction surveys. We have shown in this paper the existing work around recommendation systems, especially on collaborative filtering and machine learning, specifically on unsupervised learning.

This research is a partial contribution of my thesis which is implementation course. The first results that will be obtained will be presented in the final paper.

\section{REFERENCES}

Abdi, H. (2007). The method of least squares. Encyclopedia of Measurement and Statistics. CA, USA: Thousand Oaks.

Bouneffouf, D., Bouzeghoub, A., \& Ganarski, A. L. (2013, January). Risk-aware recommender systems. In Neural Information Processing (pp. 57-65). Springer Berlin Heidelberg.

Chen, L. S., Hsu, F. H., Chen, M. C., \& Hsu, Y. C. (2008). Developing recommender systems with the consideration of product profitability for sellers. Information Sciences, 178(4), 1032-1048

Emiliano Noce, Alberto Dellacasa Bellingegni, Anna Lisa Ciancio, Rinaldo Sacchetti, Angelo Davalli, Eugenio Guglielmelli, Loredana Zollo, EMG and ENG-envelope pattern recognition for prosthetic hand control, Journal of Neuroscience Methods, Volume 311, 2019,Pages 38-46,ISSN 0165-0270,

Ezequiel López-Rubio, Esteban J. Palomo, Francisco Ortega-Zamorano, Unsupervised learning by cluster quality optimization, Information Sciences, Volumes 436-437, 2018, Pages 31-55, ISSN 0020-0255,

Friedman, N., Geiger, D., \& Goldszmidt, M. (1997). Bayesian network classifiers. Machine learning, 29(2-3), 131-163.

Gangadhar Shobha, Shanta Rangaswamy, Chapter 8 - Machine Learning, Editor(s): Venkat N. Gudivada, C.R. Rao, Handbook of Statistics, Elsevier, Volume 38, 2018, Pages 197-228, ISSN 0169-7161, ISBN 9780444640420 ,

Hulusi Karagel, Döndü Üçeçam Karagel, Identification and Importance of Headings and Key Words in Research in the Framework of Geography Methodology, Procedia - Social and Behavioral Sciences, Volume 120, 2014, Pages 356-364, ISSN 1877-0428,

Goldberg, D., Nichols, D., Oki, B. M., \& Terry, D.(1992). Using collaborative filtering to weave an information tapestry. Communications of the ACM, 35 (12), 61-70.

Jinhong Huang, Zhu Liang Yu, Zhenghui Gu, A clustering method based on extreme learning machine, Neurocomputing,Volume 277, 2018, Pages 108-119, ISSN 0925-2312,

Joachim Schöpfel, Hélène Prost, Violane Rebouillat, Research Data in Current Research Information Systems, Procedia Computer Science, Volume 106, 2017, Pages 305-320,ISSN 1877-0509,

Liu, J., Dolan, P., \& Pedersen, E. R. (2010, February). Personalized news recommendation based on click behavior. In Proceedings of the 15th international conference on Intelligent user interfaces (pp. 31-40). ACM.

Martens, H. H. (1959). Two notes on machine "Learning". Information and Control, 2(4), 364-379.

Mohammed Tadlaoui, Sébastien George, Karim Sehaba. Approche pour la recommandation de ressources pédagogiques basée sur les liens sociaux. Sébastien George, Gaëlle Molinari, Chihab Cherkaoui, Driss Mammas et Lahcen Oubahssi. 7ème Conférence sur les Environnements Infor- matiques pour l'Apprentissage Humain (EIAH 2015), Jun 2015, Agadir, Maroc. pp.192-203, 2015.

Nynke E. Vellinga, From the testing to the deployment of self-driving cars: Legal challenges to policymakers on the road ahead, Computer Law \& Security Review, Volume 33, Issue 6,2017,Pages 847-863, ISSN 0267-3649,

Prem N. Ramkumar, Sergio M. Navarro, Heather S. Haeberle, Jaret M. Karnuta, Michael A. Mont, Joseph P. Iannotti, Brendan M. Patterson, Viktor E. Krebs, Development and Validation of a Machine Learning Algorithm After Primary Total Hip Arthroplasty: Applications to Length of Stay and Payment Models, The Journal of Arthroplasty, 2018, ISSN 0883-5403,

Samuel Eisenberg, Chapter 3 - Machine Learning for the Government: Challenges and Statistical Difficulties, Editor(s): Feras A. Batarseh, Ruixin Yang, Federal Data Science, Academic Press, 2018, Pages 29-40, ISBN 9780128124437,

Steck, H. (2013, October). Evaluation of recommendations: rating-prediction and ranking. In Proceedings of the 7th ACM Conference on Recommender Systems (pp. 213-220). ACM

Simon Willcock, Javier Martínez-López, Danny A.P. Hooftman, Kenneth J. Bagstad, Stefano Balbi, Alessia Marzo, Carlo Prato, Saverio Sciandrello, Giovanni Signorello, Brian Voigt, Ferdinando Villa, James M. Bullock, Ioannis N. Athanasiadis, Machine learning for ecosystem services, Ecosystem Services, Volume 33, Part B, 2018, Pages 165-174,ISSN 2212-0416,

Walid Cherif, Optimization of K-NN algorithm by clustering and reliability coefficients: application to breast-cancer diagnosis, Procedia Computer Science, Volume 127, 2018,Pages 293-299,ISSN 1877-0509,

Yanxin Zhang, Ye Ma, Application of supervised machine learning algorithms in the classification of sagittal gait patterns of cerebral palsy children with spastic diplegia, Computers in Biology and Medicine, Volume 106, 2019, Pages 33-39,ISSN 0010-4825,

Yong Li, Hui Huang, Validating the Job Satisfaction Survey in voluntary child welfare, Children and Youth Services Review, Volume 83, 2017, Pages 1-8, ISSN 0190-7409 